\title{
Epitaxial growth of bismuth films and bismuth-antimony heterostructures
}

\author{
J. A. van Hulst, H. M. Jaeger, ${ }^{*}$ and S. Radelaar \\ Delft Institute of Microelectronics and Submicron Technology, Delft University of Technology, Lorentzweg 1, \\ 2628 CJ Delft, The Netherlands
}

(Received 13 January 1995)

\begin{abstract}
We describe an approach for fabricating high-quality $\mathrm{Bi}$ thin films and heterostructures on $\mathrm{BaF}_{2}$ substrates by epitaxially growing them between layers of semiconducting $\mathrm{Bi}_{1-x} \mathrm{Sb}_{x}$. We present results from reflection high-energy electron diffraction, scanning electron microscopy, and atomic force microscopy analysis and show that the films are single crystalline with typical rms roughness of $1 \mathrm{~nm}$ and a dislocation density of $2 \times 10^{9} \mathrm{~cm}^{-2}$. Low-temperature magnetoresistance measurements are discussed in detail for a $90-\mathrm{nm} \mathrm{Bi}_{0.95} \mathrm{Sb}_{0.05} / 45-\mathrm{nm} \mathrm{Bi} / 65-\mathrm{nm} \mathrm{Bi}_{0.95} \mathrm{Sb}_{0.05}$ heterostructure. At liquid-helium temperatures, the electrical transport in the central, 45-nm-thick Bi layer is well described by a three-carrier model that takes into account high mobility electrons $\left(\mu_{1}=1.0 \times 10^{5} \mathrm{~cm}^{2} / \mathrm{V} \mathrm{s}\right)$ and holes $\left(\nu=3.1 \times 10^{4} \mathrm{~cm}^{2} / \mathrm{V} \mathrm{s}\right)$, as well as low mobility surface charges. The electron and hole densities are roughly equal and a factor of 6 higher than in the bulk. The epitaxial growth and clean interfaces result in a long electron elasticscattering length, $l_{\mathrm{el}}=0.38 \mu \mathrm{m}$. From an analysis of the observed Shubnikov-de Haas oscillations we obtain values for the extremal cross section of the Fermi surface, the cyclotron mass, and the singleparticle relaxation time. At $45 \mathrm{~nm}$ the film thickness is comparable to the Fermi wavelength and, due to quantum confinement, only a few two-dimensional subbands of the electron pocket are filled.
\end{abstract}

\section{INTRODUCTION}

Bismuth is a semimetal with unique electrical transport properties. These derive from a low carrier density, five orders of magnitude lower than ordinary metals, and a large Fermi wavelength, $\lambda_{F} \approx 40 \mathrm{~nm}$, that is more than a factor of 100 larger than in metals. ${ }^{1-3}$ These characteristics open up the possibility to fabricate structures that have typical sizes of the order of the Fermi wavelength, and that contain relatively few carriers. It was therefore predicted very early on that manifestations of quantum transport should be observable in $\mathrm{Bi}$. The first experiments on thickness-dependent quantum-size effects were carried out in the 1960 s in thin Bi films, and the search for a transition from semimetallic behavior to a state characterized by a band gap below some critical thickness of the order of $30 \mathrm{~nm}$ has been the focus of much theoretical and experimental work ever since. ${ }^{4-8} \mathrm{~A}$ major obstacle for the clear observation of quantum-size effects in $\mathrm{Bi}$, however, has been the unavailability of sufficiently thin films with bulklike carrier densities, mobilities, and Fermi wavelengths.

There are two main reasons for this problem. A process enabling large-scale single crystalline film growth, such as molecular-beam epitaxy, has only very recently been demonstrated for $\mathrm{Bi}$ by Partin et al. ${ }^{6}$ on $\mathrm{BaF}_{2}$ substrates, and by Hoffman et al. ${ }^{7}$ on CdTe substrates. In addition, Komnik et al. ${ }^{8}$ pointed out in 1971 that in thin films band bending at the interface with the substrate or the free surface leads to additional surface states. This might increase the actual carrier density in thin films over the bulk value, and thus reduce the Fermi wavelength.

Here we describe an alternative approach, using a semiconducting $\mathrm{Bi}_{1-x} \mathrm{Sb}_{x}$ buffer layer grown on a $\mathrm{BaF}_{2}$ substrate. The top of the buffer is single crystalline and serves as a substrate for the subsequent growth of a partially relaxed epitaxial $\mathrm{Bi}$ layer. The structure is capped with a second, protective layer of partially relaxed $\mathrm{Bi}_{1-x} \mathrm{Sb}_{x}$. We find that this technique can be extended to multilayer structures without loss of epitaxy. Sandwiching the $\mathrm{Bi}$ layer between epitaxial semiconducting $\mathrm{Bi}_{1-x} \mathrm{Sb}_{x}$ has several advantages. First, the charge carriers in the $\mathrm{Bi}$ layer have a large wavelength and experience mostly specular reflection at a sharp $\mathrm{Bi} / \mathrm{Bi}_{1-x} \mathrm{Sb}_{x}$ interface, enhancing the overall mobility. Second, the band structure of $\mathrm{Bi}_{1-x} \mathrm{Sb}_{x}$ is only slightly different from that of $\mathrm{Bi}$, so one expects only a small band bending at the interface. This is in contrast to the band bending at a $\mathrm{Bi} /$ vacuum interface, which can increase the chargecarrier density by more than one order of magnitude, ${ }^{9}$ and therefore diminish any quantum-size effects (QSE's). Finally, the parallel conductance from semiconducting $\mathrm{Bi}_{1-x} \mathrm{Sb}_{x}$ layers freezes out at liquid-helium temperatures.

Bismuth layers usually have a (111) texture, suggesting the use of a substrate commensurate with the (111) plane. In the literature one mainly finds two substrate choices: mica ${ }^{5,10-12}$ and $\mathrm{BaF}_{2}(111) .^{6,13-15}$ On mica, Jing et al. ${ }^{12}$ found a growth mechanism characterized by initial nucleation and formation of small islands, followed by layer-by-layer growth of each island, and finally coalescence of the islands. The problem with mica is twinning, because two different growth orientations are equally probable. As a result, Bi films on mica are composed of two crystal orientations and contain twin boundaries. Partin et al. ${ }^{6}$ circumvented this problem by using the molecular-beam epitaxy (MBE) of $\mathrm{Bi}$ on $\mathrm{BaF}_{2}$ substrates. They were able to grow thick $\left(d_{\mathrm{Bi}} \gg \lambda_{\mathrm{F}}\right)$ single crystalline Bi films and, to our knowledge, for the first time in a 
Bi film, observe Shubnikov-de Haas oscillations. However, even with the method developed by Partin et al. the first $90 \mathrm{~nm}$ of a film were found to be polycrystalline. ${ }^{16}$

$\mathrm{Bi}$ has a rhombohedral crystal structure that can be visualized as a fcc crystal stretched along the body diagonal. This distortion causes an overlap of $38 \mathrm{meV}$ between the valence band at point $T$ in reciprocal space and the conduction bands at the $L$ points (Fig. 1). The addition of $\mathrm{Sb}$ to $\mathrm{Bi}$ changes the lattice parameters to values given (in hexagonal coordinates) by $a=(4.546-x 0.2384) \AA$ and $c=(11.863-x 0.5166) \AA$ at $T=298 \mathrm{~K} .{ }^{17}$ Here $x$ is the fraction of $\mathrm{Bi}$ atoms substituted by $\mathrm{Sb}$. Moreover, the band structure changes. For $0 \leq x<0.06$ bulk $\mathrm{Bi}_{1-x} \mathrm{Sb}_{x}$ remains a semimetal with, however, a decreasing band overlap for increasing $x$. In the range $0.06 \leq x \leq 0.4$ bulk $\mathrm{Bi}_{1-x} \mathrm{Sb}_{x}$ becomes a semiconductor, with its maximum gap of $14 \mathrm{meV}$ at $x=0.12 .^{18-20}$

In a $\mathrm{Bi} / \mathrm{Bi}_{1-x} \mathrm{Sb}_{x}$ heterostructure any difference in lattice parameter $a$ will cause stresses. As a result, $\mathrm{Bi}_{1-x} \mathrm{Sb}_{x}$ will be under tensile stress and $\mathrm{Bi}$ under compressive stress, which changes the overlap between electron and hole pockets. ${ }^{21}$ Relaxation of these stresses creates dislocations, and decreases the $\mathrm{Bi}$ transport mobility. Since a small overlap and a large mobility are desirable, it becomes important to choose a small Sb concentration, just sufficient to reduce the low-temperature parallel conductance of the Sb-containing layers compared to that of the enclosed Bi film. Our results show that for $\mathrm{Bi}_{1-x} \mathrm{Sb}_{x}$ films semiconducting behavior is still observed at $\mathrm{Sb}$ concentrations as small as $x=0.05$.

This paper is organized as follows: In Sec. II we describe the growth and the structural characterization of $\mathrm{Bi}_{1-x} \mathrm{Sb}_{x}$ films and heterostructures. For the discussion of the resulting electrical transport properties, in Secs. III and IV, we focus mainly on measurements performed on a $\mathrm{Bi} / \mathrm{Bi}_{0.95} \mathrm{Sb}_{0.05}$ heterostructure that encloses a central, 45-nm-thick $\mathrm{Bi}$ layer. In Sec. III we present lowtemperature conductance measurements in fields up to 13

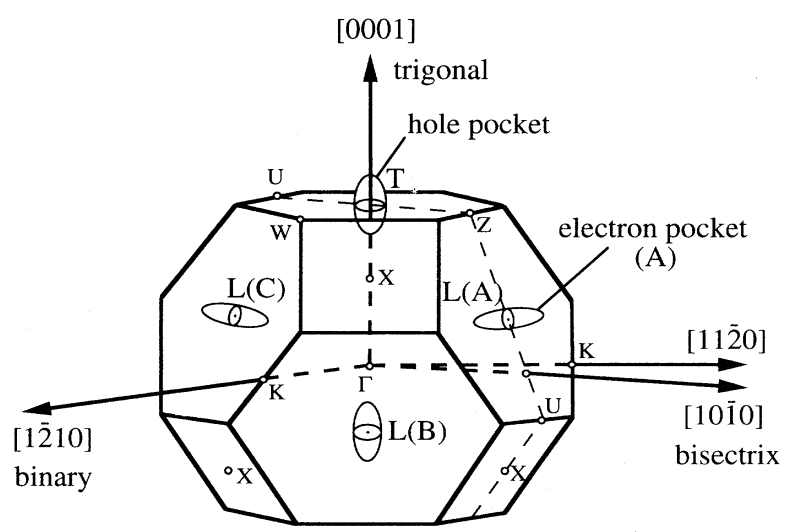

FIG. 1. Perspective view of the Brillouin zone of bismuth. The trigonal direction is the growth direction perpendicular to the substrate, the binary and bisectrix are in the film plane. The electron pockets at the $L$ points have threefold symmetry with respect to the trigonal axis. The hole pocket at point $T$ has rotational symmetry around the trigonal axis. After Ref. 33.
T. From fits to the magnetoconductance data we obtain the carrier densities, mobilities, and elastic-scattering times. The high sample quality resulting from our growth process allows for a clean observation of Shubnikov-de Haas oscillations from which we deduce the extremal cross section of the Fermi surface, the cyclotron mass, and the single-particle relaxation time. Section IV discusses these measurements and compares our fitting model to other approaches used in the literature. In the concluding Sec. V we summarize our results and point to possible extensions of our growth technique.

\section{SAMPLE GROWTH}

All films were grown in an ultrahigh-vacuum chamber, equipped with an in situ reflection high-energy electrondiffraction (RHEED) system. The chamber was pumped by titanium sublimation and ion getter pumps, resulting in a base pressure of $1 \times 10^{-11}$ Torr. During growth, cryopanels kept the pressure below $3 \times 10^{-11}$ Torr. Bi $(99.9999 \%)$ and $\mathrm{Sb}(99.9999 \%)$ were evaporated from Knudsen cells under shutter control, and a mass spectrometer was used to measure both semimetal fluxes. For example, at cell temperatures of $606^{\circ} \mathrm{C}$ for $\mathrm{Bi}$ and $408^{\circ} \mathrm{C}$ for $\mathrm{Sb}$, we were able to grow $\mathrm{Bi}_{0.95} \mathrm{Sb}_{0.05}$ at a rate of 466 $\mathrm{nm} / \mathrm{h}$ as calibrated by weight measurements of the sample before and after growth.

Substrates of $10 \times 10 \times 1.5 \mathrm{~mm}^{3}$ were cleaved from a $\mathrm{BaF}_{2}$ single crystal. ${ }^{22}$ The natural (111) cleavage plane in $\mathrm{BaF}_{2}$ has a misfit of $-3.6 \%$ with the $\mathrm{Bi}(111)$ plane. The cleavage plane is atomically flat on a microscopic scale, but has some steps of several $\mu \mathrm{m}$ on a mm length scale. ${ }^{23}$ Dislocations in $\mathrm{BaF}_{2}$ can easily be made visible in an optical microscope by first exposing the crystal surface to water. For our substrate crystal we determined a dislocation density of $(20 \pm 6) \times 10^{4} \mathrm{~cm}^{-2}$. Before the film growth the substrate was heated for several minutes both in the introduction chamber and the growth chamber (at 550 and $450^{\circ} \mathrm{C}$, respectively).

For heterostructures and multilayers, growth was started in each case with a $90 \mathrm{~nm}$-thick $\mathrm{Bi}_{1-x} \mathrm{Sb}_{x}$ buffer layer. This layer was single crystalline on its top surface, even though polycrystalline growth can occur in the first stages of growth. ${ }^{16}$ On this buffer layer, Bi films could be grown epitaxially, and were finally capped with another $\mathrm{Bi}_{1-x} \mathrm{Sb}_{x}$ layer. This guaranteed clean interfaces and prevented exposure of the Bi film to air. During growth a substrate temperature of $70^{\circ} \mathrm{C}$ was chosen, the lowest temperature still resulting in single crystalline growth and smooth film surfaces, yet preventing significant interdiffusion of $\mathrm{Bi}$ and $\mathrm{Sb}$.

To find this optimal growth temperature, we investigated a series of pure $\mathrm{Bi}$ films of $500-\mathrm{nm}$ thickness. RHEED images showed single crystalline growth at substrate temperatures of 50,80 , and $110^{\circ} \mathrm{C}$ (samples $a, b, c$, and $d$; see Fig. 2). ${ }^{24}$ The diffraction pattern exhibited two-dimensional (2D) character (lines), with only a small additional 3D component (spots), indicative of a very smooth surface. At a substrate temperature of $20^{\circ} \mathrm{C}$ (sample $e$ ), on the other hand, the 3D RHEED pattern of a rough sample surface was observed. $\mathrm{As} \mathrm{Sb}$ is added to 


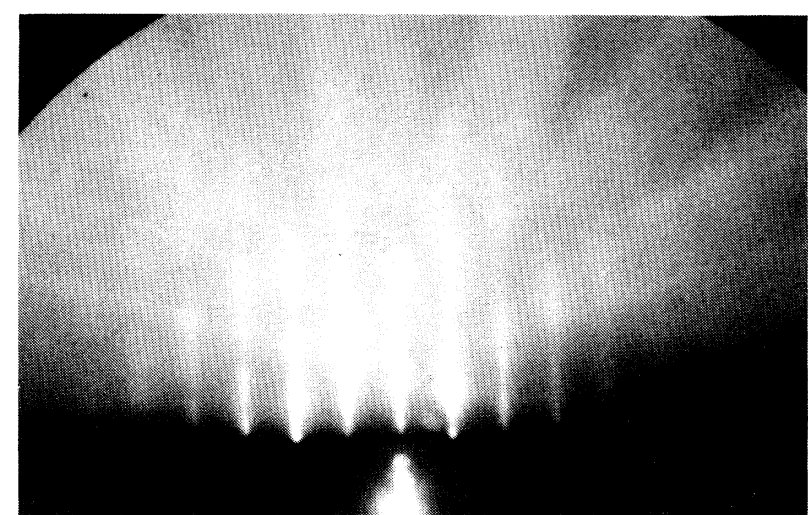

FIG. 2. RHEED pattern of a 675-nm Bi(111) film grown at $110^{\circ} \mathrm{C}$ (sample $c$ ). The electron beam had an energy of $50 \mathrm{keV}$ and a $(\overline{1}, 0,1)$ azimuth orientation. The diffraction pattern exhibits 2D character (lines) with only a small additional 3D component (spots), indicative of a very smooth surface.

$\mathrm{Bi}$, the surface mobility during growth is reduced as compared to the pure $\mathrm{Bi}$ case. For this reason we found that single crystalline growth of $\mathrm{Bi}_{1-x} \mathrm{Sb}_{x}(x<0.16)$ was only possible at substrate temperatures of $70^{\circ} \mathrm{C}$ or above. In situ RHEED during the growth of a heterostructure shows comparable and constant pictures for $\mathrm{Bi}$ and $\mathrm{BiSb}$ layers. The crystal properties and roughness at the surface are representative of the crystal structure of the $\mathrm{Bi}$ layer and the roughness of the $\mathrm{Bi} / \mathrm{BiSb}$ interface. Figure 3 shows a scanning-electron-microscope (SEM) picture of a 200-nm-thick heterostructure (sample $f$; thickness of the enclosed $\mathrm{Bi}$ film $d_{\mathrm{Bi}}=45 \mathrm{~nm}$ ), tilted at an angle of $10^{\circ}$. Atomic-force-microscope (AFM) measurements show that the rms roughness of the film is about $1 \mathrm{~nm}$.

A selective etch (in $\mathrm{HNO}_{3}+2$-propanol $+\mathrm{H}_{2} \mathrm{O}$ at a ratio of $1: 5: 4$ by volume ${ }^{25}$ ) was used to determine the dislocation density. Figure 4 shows an AFM image of the heterostructure, taken after a 1-min etch at room temperature. The scan field is $2 \times 2 \mu \mathrm{m}^{2}$, and is representative of the whole film. One can see clearly that all etch pits have the same orientation, indicating that the heterostructure is indeed single crystalline. Measurement of the density

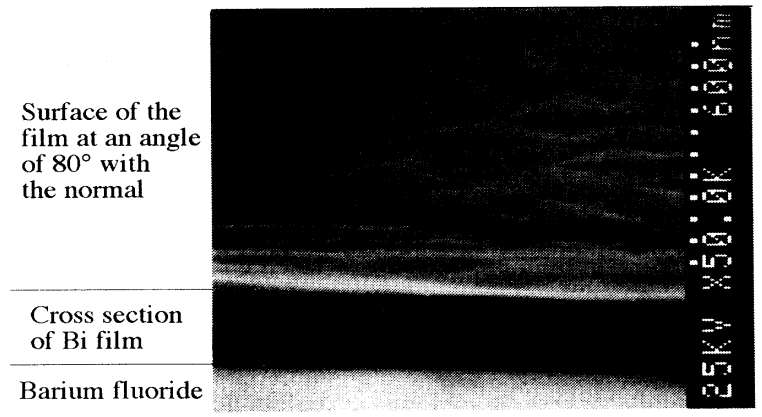

FIG. 3. Scanning-electron-microscope (SEM) image of a 200-nm-thick heterostructure (sample $f$ ). A cross section of the heterostructure is seen in the foreground, with very little surface roughness visible at the top (magnification $50000 \times$ at a tilt angle of $80^{\circ}$ with respect to the surface normal).

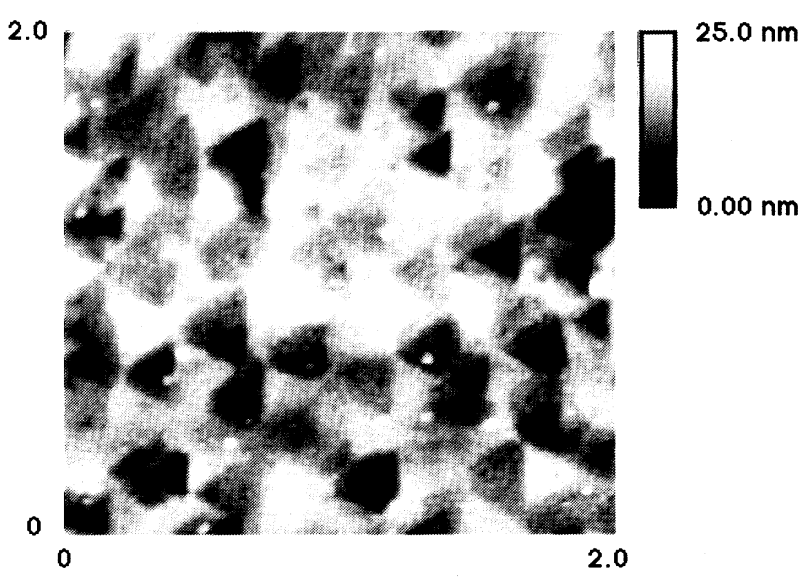

FIG. 4. Atomic-force-microscope (AFM) image of the sample sample as in Fig. 3 (sample $f$ ) after a selective etch for dislocations. The scan field is $2 \times 2 \mu \mathrm{m}^{2}$ and is representative of the whole film. All triangular pits exhibit the same orientation, indicating that the sample is single crystalline. The etch pit density corresponds to $2 \times 10^{9}$ dislocations per $\mathrm{cm}^{2}$.

of etch pits gave a value of typically $2 \times 10^{9}$ interface dislocations per $\mathrm{cm}^{2}$.

Table I lists the growth characteristics for three types of structures: $\mathrm{Bi}$ films, $\mathrm{Bi}_{1-x} \mathrm{Sb}_{x}$ films, and $\mathrm{Bi} / \mathrm{Bi}_{1-x} \mathrm{Sb}_{x}$ heterostructures.

\section{TRANSPORT MEASUREMENTS AND ANALYSIS}

For the low-temperature electronic transport characterization a helium flow cryostat was used with a temperature control between $1.2 \mathrm{~K}$ and room temperature, and magnetic fields up to $14 \mathrm{~T}$. The sample resistance was measured in a four-terminal configuration with a Linear Research LR400 resistance bridge.

Figure 5 compares resistance vs temperature measurements on sample $f$, the $\mathrm{Bi} / \mathrm{Bi}_{0.95} \mathrm{Sb}_{0.05}$ heterostructure with the enclosed 45-nm Bi layer discussed in Sec. II, to results obtained on thicker films of pure Bi (sample $b$ ) and $\mathrm{Bi}_{0.95} \mathrm{Sb}_{0.05}$ (sample $g$ ). Although the concentration $x=0.05$ in the two Sb-containing samples is just below the literature value $x=0.06$, for the onset of semicon-

TABLE I. Overview of samples discussed in this paper. $T_{\text {sub }}$ is the substrate temperature during growth. The last column describes the thickness and composition.

\begin{tabular}{llrr}
\hline \hline $\begin{array}{l}\text { Sample } \\
\text { number }\end{array}$ & $\begin{array}{c}\text { Kind } \\
\text { of }\end{array}$ & & \\
\hline$a$ & ftructure & $T_{\text {sub }}\left({ }^{\circ} \mathrm{C}\right)$ & Description \\
$b$ & film & 50 & $500-\mathrm{nm} \mathrm{Bi}$ \\
$c$ & film & 80 & $500-\mathrm{nm} \mathrm{Bi}$ \\
$d$ & film & 110 & $675-\mathrm{nm} \mathrm{Bi}$ \\
$e$ & film & 110 & $500-\mathrm{nm} \mathrm{Bi}$ \\
$f$ & heterostructure & 20 & $500-\mathrm{nm} \mathrm{Bi}$ \\
& & 710 & $90-\mathrm{nm} \mathrm{Bi}{ }_{0.95} \mathrm{Sb}_{0.05}$ \\
$g$ & film & 110 & $45-\mathrm{nm} \mathrm{Bi} / 65-\mathrm{nm} \mathrm{Bi} \mathrm{Bi}_{0.95} \mathrm{Sb}_{0.05}$ \\
$h$ & heterostructure & 50 & $450-\mathrm{nm} \mathrm{Bi} \mathrm{Bi}_{0.95} \mathrm{Sb}_{0.05}$ \\
& & & $50-\mathrm{nm} \mathrm{Bi} \mathrm{Bi}_{0.84} \mathrm{Sb}_{0.16}$ \\
\hline \hline
\end{tabular}




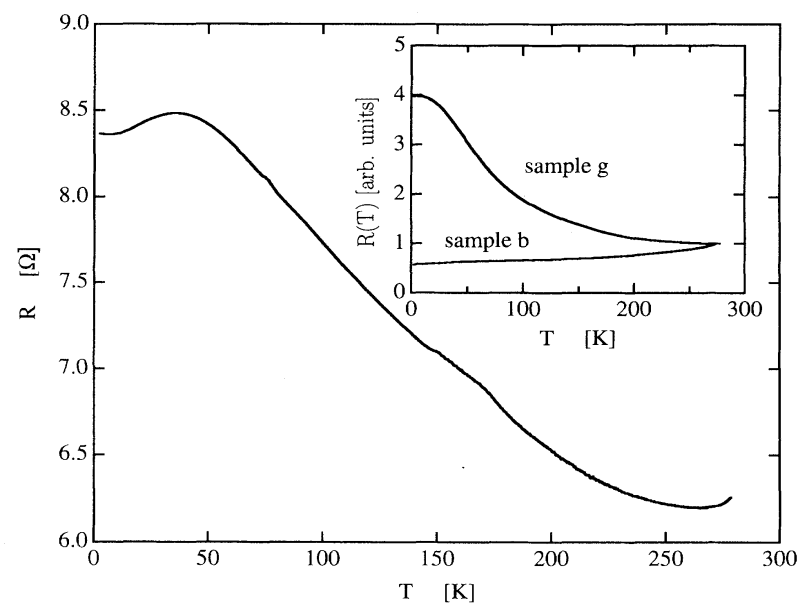

FIG. 5. Resistance as a function of temperature, $R(T)$, for a 90-nm $\mathrm{Bi}_{0.95} \mathrm{Sb}_{0.05} / 45-\mathrm{nm} \mathrm{Bi} / 65-\mathrm{nm} \mathrm{Bi}_{0.95} \mathrm{Sb}_{0.05}$ heterostructure (sample $f$ ). The inset shows $R(T)$ curves for a 500-nm-thick pure $\mathrm{Bi}$ film (sample $b$ ) and for a $450-\mathrm{nm}$-thick $\mathrm{Bi}_{0.95} \mathrm{Sb}_{0.05}$ film (sample $g$ ).

ducting behavior in bulk the inset in Fig. 5 clearly shows that even in a $450-\mathrm{nm}$-thick $\mathrm{Bi}_{0.95} \mathrm{Sb}_{0.05}$ film the resistance increases strongly with decreasing temperature. In contrast, the 500-nm-thick pure Bi film exhibits a decreasing resistance with temperature. The resistance trace shown in the main part of Fig. 5 for the heterostructure can therefore be understood as resulting from the contributions of the central $\mathrm{Bi}$ layer and the two outer $\mathrm{Bi}_{0.95} \mathrm{Sb}_{0.05}$ layers connected in parallel.

In the following, we limit our discussion to results obtained from the $\mathrm{Bi} / \mathrm{Bi}_{0.95} \mathrm{Sb}_{0.05}$ heterostructure (sample $f$ ). Data consistent with these results were obtained from measurements on several similar heterostructures.

Resistance measurements were taken for positive and negative magnetic field $B$, perpendicular to the substrate. From the symmetric and asymmetric contributions with respect to zero field the magnetoresistance and Hall resistance, respectively, were calculated. Figure 6 shows the (sheet) magnetoconductance $g_{x x}(B)$ and the Hall conductance $g_{x y}(B)$ for a magnetic field perpendicular to the substrate, taken at a temperature of $1.5 \mathrm{~K}$ (solid lines). Note the saturation of the magnetoconductance at high magnetic fields. This residual conductance is caused by low mobility carriers. The inset of Fig. 6 shows that the residual conductance appears to be independent of the film type or thickness, and was observed to a nearly identical level in very different films, such as a $500-\mathrm{nm}$ Bi film and a $450-\mathrm{nm} \mathrm{Bi}_{0.95} \mathrm{Sb}_{0.05}$ film. The existence of this residual conductance makes it necessary to include an additional term in the description of $g_{x x}(B)$ and $g_{x y}(B)$ because the usual contributions, based on the bulk conductivity tensor, vanish in the high-field limit. In converging the wide field range $0 \leq B \leq 13 \mathrm{~T}$ we find that a satisfactory fit requires that the additional term contains a small, but significant, magnetic-field dependence. Taking into account the anisotropic electron and isotropic hole contributions known from bulk $\mathrm{Bi}^{26}$ we thus arrive at the following fitting forms for the conductances:

$$
\begin{aligned}
g_{x x}(B)= & \frac{N_{2} e\left(\frac{\mu_{1}+\mu_{2}}{2}\right)}{1+\mu_{1} \mu_{2} B^{2}}+\frac{P_{2} e v}{1+v^{2} B^{2}} \\
& +\frac{P_{2, \mathrm{par}}(1-\alpha B) e v_{\mathrm{par}}}{1+v_{\mathrm{par}}^{2} B^{2}}, \\
g_{x y}(B)= & -\frac{N_{2} e \mu_{1} \mu_{2} B}{1+\mu_{1} \mu_{2} B^{2}}+\frac{P_{2} e v^{2} B}{1+v^{2} B^{2}} \\
& +\frac{P_{2, \mathrm{par}}(1-\alpha B) e v_{\mathrm{par}}^{2} B}{1+v_{\mathrm{par}}^{2} B^{2}} .
\end{aligned}
$$

Here the first two terms come from the usual conduc-
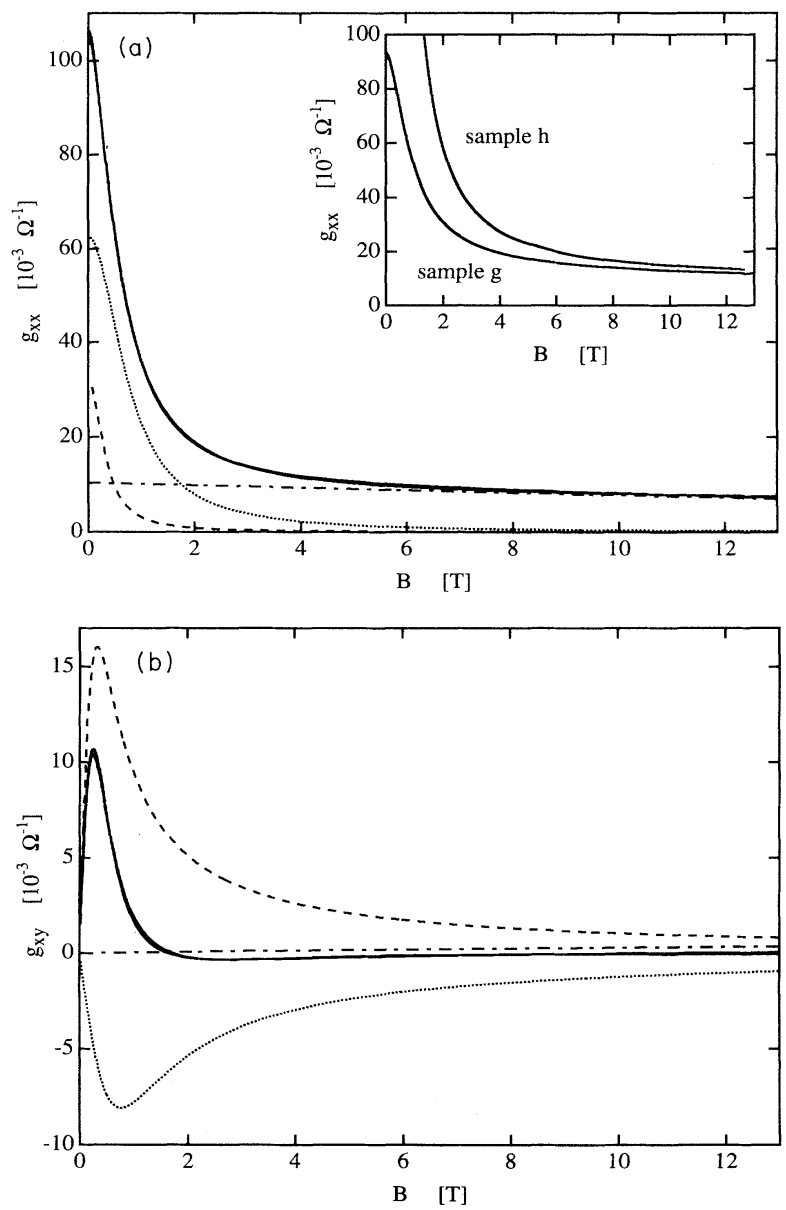

FIG. 6. (a) Longitudinal magnetoconductance $g_{x x}(B)$, and (b) Hall conductance $g_{x y}(B)$ of the heterostructure sample $f$. Also shown are the fitting results to Eqs. (1) (solid lines). On the scale of these figures, the fits cannot be distinguished from the data. To indicate their relative strength and field dependence, the individual contributions to the fit are also shown separately: electron pockets (dotted lines), hole pocket (dashed lines), and residual parallel conducting channel (dashed-dotted lines). The inset to (a) compares the magnetoconductance of a $50-\mathrm{nm}$ $\mathrm{Bi}_{0.84} \mathrm{Sb}_{0.16} / 500-\mathrm{nm} \mathrm{Bi} / 50-\mathrm{nm} \mathrm{Bi} \mathrm{Bi}_{0.84} \mathrm{Sb}_{0.16}$ (sample $h$ ) and a 450$\mathrm{nm} \mathrm{Bi}_{0.95} \mathrm{Sb}_{0.05}$ film (sample $\mathrm{g}$ ). The same level of residual conductance at high fields is also present in these films. 
tance tensor in the presence of a magnetic field. $N_{2}$ and $P_{2}$ are the sheet densities of electrons and holes, $\mu_{1}$ and $\mu_{2}$ are the (anisotropic) transport mobilities of the electrons in the binary and the bisectrix directions, and $v$ is the (isotropic) transport mobility of the holes. The third term, with the denominator $P_{2, \text { par }}(1-\alpha B)$, describes a field-dependent sheet density of holes, where $v_{\text {par }}$ is the transport mobility of these holes. We found that $g_{x x}(B)$ in the high-field limit $(B>6 \mathrm{~T})$ was very sensitive to small variations in $\alpha$ and in the product $\left(P_{2, \mathrm{par}} v_{\mathrm{par}}\right)$. These parameters were therefore optimized first (by a least-squares method), and then fixed in the further fitting. The remaining transport parameters were obtained from a simultaneous least-squares fit of $g_{x x}(B)$ and $g_{x y}(B)$. The fitting results are listed in Table II and compared to the data in Fig. 6. In this figure the contributions of each of the three terms in Eqs. (1) are also shown individually.

Superimposed on the measured longitudinal magnetoresistance $R_{x x}(B)$, we find Shubnikov-de Haas (SdH) oscillations. From work on GaAs heterostructures ${ }^{27-29}$ it is known that the magnitude of $\mathrm{SdH}$ oscillations is severely reduced by the presence of any parallel (residual) conductance channel. To better visualize the oscillations, it is convenient to plot the normalized derivative of the magnetoresistance:

$$
\frac{B}{R(B)} \frac{\delta R(B)}{\delta B}=-\frac{B}{g_{x x}(B)} \frac{\delta g_{x x}(B)}{\delta B} .
$$

This relation holds under the assumption that the Hall resistance is much smaller than the magnetoresistance, which is justified in our samples. Figures 7(a) and 7(b) show the SdH oscillations for magnetic fields perpendicular and parallel to the film and over a range of temperatures. We note that for parallel field oscillations appear only for $B \geq 1 \mathrm{~T}$, and that at higher temperatures (up to $T=70 \mathrm{~K}$ ) there is a small shift in peak positions.

In Eq. (1) there are three terms contributing to the magnetoconductance. However, only carriers with a sufficiently high mobility and a closed cyclotron orbit contribute to the SdH oscillations. Figures 7(a) and 7(b) clearly exhibit one dominant oscillation period. If we

TABLE II. Electrical characteristics of a 90-nm $\mathrm{Bi}_{0.95} \mathrm{Sb}_{0.05} / 45-\mathrm{nm} \mathrm{Bi} / 65-\mathrm{nm} \mathrm{Bi}_{0.95} \mathrm{Sb}_{0.05}$ heterostructure (sample $f$ ) at $1.5 \mathrm{~K}$. The listed values for the electron (hole; parallel conductance channel) sheet densities $N_{2}\left(P_{2} ; P_{2, \text { par }}\right)$, and mobilities $\mu_{1}$ and $\mu_{2}\left(v ; v_{\mathrm{par}}\right)$ are obtained from fits to the data in Figs. $6(\mathrm{a})$ and $6(\mathrm{~b})$ as explained in the text.

\begin{tabular}{lllc}
\hline \hline Electron pocket & $N_{2}\left(10^{12} \mathrm{~cm}^{-2}\right)$ & 7.7 & $( \pm 0.8)$ \\
& $\mu_{1}\left(10^{4} \mathrm{~cm}^{2} / \mathrm{V} \mathrm{s}\right)$ & 9.9 & $( \pm 1.0)$ \\
& $\mu_{2}\left(10^{4} \mathrm{~cm}^{2} / \mathrm{V} \mathrm{s}\right)$ & 0.17 & $( \pm 0.02)$ \\
Hole pocket & $P_{2}\left(10^{12} \mathrm{~cm}^{-2}\right)$ & 6.5 & $( \pm 0.7)$ \\
& $v\left(10^{4} \mathrm{~cm}^{2} / \mathrm{V} \mathrm{s}\right)$ & 3.1 & $( \pm 0.3)$ \\
Parallel conductance & $P_{2, \text { par }}\left(10^{15} \mathrm{~cm}^{-2}\right)$ & 1.7 & $( \pm 0.9)$ \\
& $\alpha\left(T^{-1}\right)$ & 0.025 & $( \pm 0.003)$ \\
& $v_{\text {par }}\left(\mathrm{cm}^{2} / \mathrm{V} \mathrm{s}\right)$ & 38 & $( \pm 20)$ \\
\hline \hline
\end{tabular}

therefore assume that only one term in Eq. (1) contributes to $\mathrm{SdH}$ oscillations, we can write ${ }^{30}$

$$
\begin{aligned}
g_{x x}(B)= & g_{x x, 1}(B)[1-A(B)]+g_{x x, 2}(B)+g_{x x, 3}(B), \\
A(B)= & C\left[\frac{B}{F}\right]^{1 / 2} \frac{X}{\sinh (X)} \exp \left[\frac{-\pi m_{c}\left(E_{F}\right)}{e \tau_{s} B}\right] \\
& \times \cos \left(2 \pi \frac{F}{B}+\Phi\right),
\end{aligned}
$$

where

$$
X=\frac{2 \pi^{2} k_{B} m_{c}\left(E_{F}\right) T}{\hbar e B} .
$$
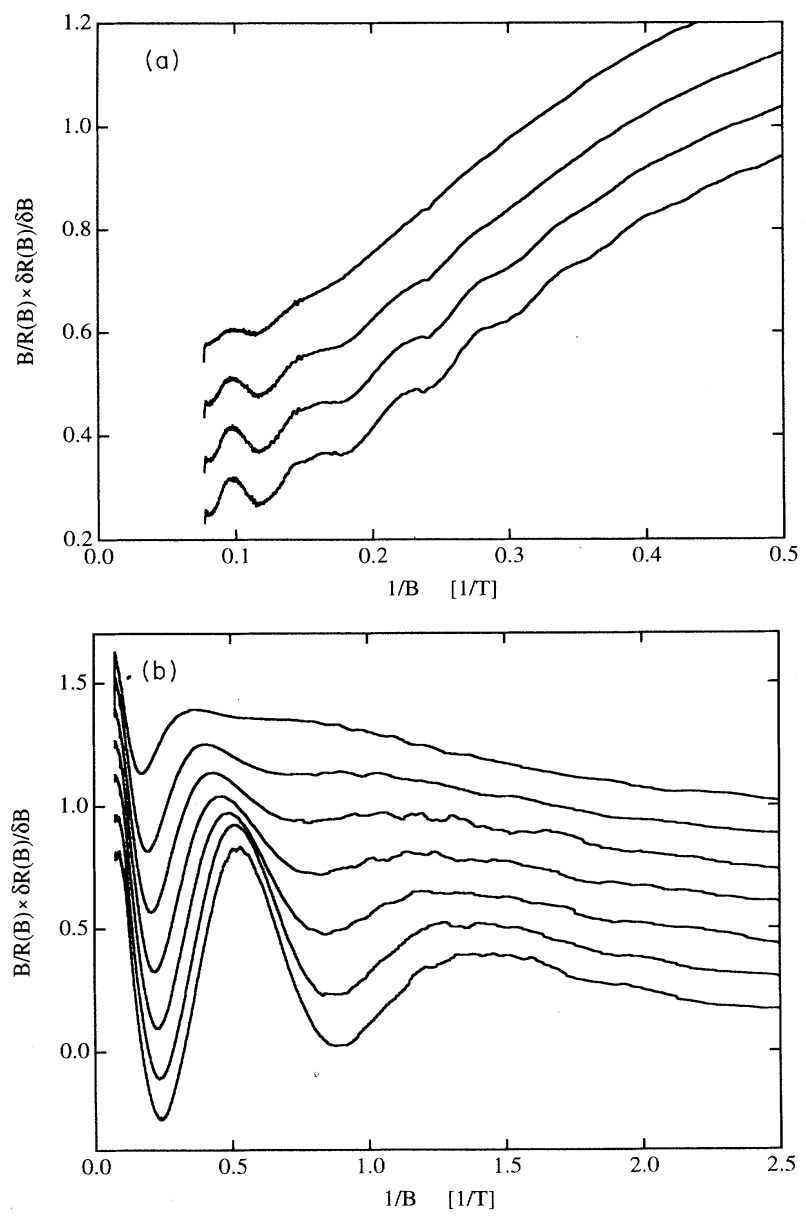

FIG. 7. (a) Shubnikov-de Haas (SdH) oscillations for a magnetic field applied perpendicular to the film plane. Shown is the dimensionless, normalized derivative of the magnetoresistance as a function of the inverse magnetic field. The traces were taken at $1.5,4.5,10$, and $20 \mathrm{~K}$ (bottom to top) and have been shifted up vertically by 0.1 with respect to the next-lower temperature. (b) The same plot but for a parallel magnetic field. The traces show SdH oscillations at temperatures 1.5, 10, 20, 30, 40, 50 , and $70 \mathrm{~K}$ (bottom to top), shifted vertically by 0.15 each. Note that there are only oscillations for magnetic fields larger than about $1 \mathrm{~T}$, and that with increasing temperatures there is a small decrease in oscillation period (see Fig. 8). 
The quantum correction $A(B)$ gives rise to $\mathrm{SdH}$ oscillations in $1 / B$ with period $1 / F$. Here $\tau_{s}$ is the singleparticle relaxation time due to scattering, ${ }^{31} \Phi$ an arbitrary phase factor, and $C$ a constant of order unity. $F$ is a measure of the extremal cross section through the Fermi surface perpendicular to the direction of the magnetic field.

At $T=1.5 \mathrm{~K}$, and over the magnetic-field range shown in Fig. 7, the term $X / \sinh X$ is very close to unity. The oscillation amplitude in $-\left[B / g_{x x}(B)\right]\left[\delta g_{x x}(B) / \delta B\right]$ is then given by

$$
\begin{aligned}
\frac{g_{x x, 1}(B)}{g_{x x}(B)} C & {\left[\frac{B}{F}\right]^{1 / 2} \exp \left[\frac{-\pi m_{c}\left(E_{F}\right)}{e \tau_{s} B}\right] } \\
& \times\left[\left(2 \pi \frac{F}{B}\right]^{2}+\left[\frac{1}{2}+\frac{\pi m_{c}\left(E_{F}\right)}{e \tau_{s} B}\right]^{2}\right]^{1 / 2} .
\end{aligned}
$$

This formula is used to fit the quotient $m_{c}\left(E_{F}\right) / \tau_{s}$. Similarly, a fit at fixed $B$ yields the cyclotron mass $m_{c}\left(E_{F}\right)$. For magnetic fields perpendicular to the film or heterostructure, the factor $g_{x x, 1}(B) / g_{x x}(B)$ is known for both electrons $\left(N_{2}\right)$ and holes $\left(P_{2}\right)$. A fit of $\tau_{s}$ and $m_{c}\left(E_{F}\right)$ was made for both cases and, within the error margins, shows comparable results. For $B$ parallel to the film, we set this factor to be equal to 1 , and the result of the fit to Eq. (4) is an estimate for the order of magnitude of $\tau_{s}$ and $m_{c}\left(E_{F}\right)$. The results are shown in Table III.

We now need to address the question of whether the SdH oscillations are caused by electrons or holes, i.e., whether the first term for the magnetoconductance in Eq. (3) corresponds to the electron or hole term in Eq. (1). The bulk Bi values for $F$ and $m_{c}\left(E_{F}\right)$ (Refs. 1 and 5) may be a guideline: For a $B$ field along the trigonal axis, i.e., perpendicular to the film plane, electrons and holes have comparable values for $F$ and $m_{c}\left(E_{F}\right)$. All three electron pockets give $F=6.4 \mathrm{~T}$, corresponding to a cross section at $k_{z}=0$. For a parallel $B$ field there is always one electron pocket whose bisectrix makes only a small angle $\left(\leq 30^{\circ}\right)$ with the $B$ field. This pocket will dominate the SdH oscillations with its large period $1 / F$. To obtain an estimate for this period we assume a $B$ field along the

TABLE III. Fitting results from the analysis of Shubnikov-de Haas (SdH) oscillations measured in a heterostructure with 45 -nm-thick central Bi layer (sample $f$ ). The corresponding measured magnetoconductance data are shown in Figs. $7(\mathrm{a})$ and $7(\mathrm{~b}) . \quad F$ is the inverse of the $\mathrm{SdH}$ oscillation period, $m_{c}\left(E_{F}\right)$ is the cyclotron mass at the Fermi energy, $m_{0}$ is the free-electron mass, and $\tau_{s}$ is the single-particle relaxation time. The subscripts 1 or $\|$ denote the applied magnetic field perpendicular or parallel to the film plane.

\begin{tabular}{lcc}
\hline \hline$F_{\perp}(T)$ & 17 & $( \pm 2)$ \\
$F_{\|}(T)$ & 1.6 & $( \pm 0.05)$ \\
& & $( \pm 0.01)$ \\
$m_{c \perp}\left(E_{F}\right)\left(m_{0}\right)$ & 0.10 & $( \pm 0.001)$ \\
$m_{c \|}\left(E_{F}\right)\left(m_{0}\right)$ & 0.009 & \\
& & $( \pm 0.008)$ \\
$\tau_{s \perp}(\mathrm{ps})$ & 0.085 & $( \pm 0.008)$ \\
$\tau_{s \|}(\mathrm{ps})$ & 0.074 & \\
\hline
\end{tabular}

bisectrix. This leads to $F \approx 1.2 \mathrm{~T}$. For convenience, the various values for $F$ are listed in Table IV.

Comparing the values obtained form fits to the experimental data (Table III) with the values expected from the theoretical considerations outlined above (Table IV), we conclude that in a parallel magnetic field the electron pockets cause the $\mathrm{SdH}$ oscillations. For the perpendicular $B$ field, we cannot distinguish between electron or hole pockets as the source of the $\mathbf{S d H}$ oscillations, although holes are the more likely candidate because of their higher transport mobility $\left(v^{2}>\mu_{1} \mu_{2}\right)$.

From the fitting results for the mobilities $\mu_{1}, \mu_{2}$, and $v$ (Table II), and using the mass tensor for electrons and holes, ${ }^{1,5}$ the elastic-scattering times can be calculated (Table V). For electrons in the binary (bisectrix) directions we obtain $\tau_{t, e 1}=0.47 \mathrm{ps}\left(\tau_{t, e 2}=1.9 \mathrm{ps}\right)$, while holes in the film plane have $\tau_{t, h}=1.1 \mathrm{ps}$. The corresponding mean free paths can be calculated from the mobilities and dimensions of the electron ellipses. The resulting values for $1_{e 1}=0.38 \mu \mathrm{m}$ and $1_{h}=0.5 \mu \mathrm{m}$ are remarkable if we compare them to the $45 \mathrm{~nm}$ Bi-layer thickness.

\section{DISCUSSION}

The model we use for the description of $g_{x x}(B)$ and $g_{x y}(B)$ can be divided into two parts. The first part contains two terms for the high mobility channels of electrons and holes, and the second part a term for a low mobility parallel (residual) conductance channel. The first part is similar to models for Bi previously introduced by Hartman ${ }^{26}$ and Chu, ${ }^{5}$ but goes beyond these in several aspects. Specifically, Eq. (1) is valid up to large $B$ fields, while the in-plane conductivities obtainable from the three-dimensional model for bulk bismuth by Hartman are appropriate only for the limit of small magnetic fields since he expands the conductivity tensor to lowest order in $B$. Chu's two-dimensional model $a$ priori introduces the charge neutrality condition $\left(N_{2}=P_{2}\right)$, and also assumes a homogeneous lifetime for electrons $\tau_{e}$, which results in a constant relationship between $\mu_{1}$ and $\mu_{2}$.

The data in Fig. 6 can only be described well after the introduction of an additional term to the magnetoconductance, corresponding to a parallel channel of low mobility holes. Because our measurements on completely different films exhibit the same amount of parallel conductance, independent of the bismuth film thickness and independent of antimony concentration, we believe that these additional holes are localized at the interfaces. The large sheet density of such holes obtained from the fits,

TABLE IV. The values for $m_{c}\left(E_{F}\right)$ and $F$ in bulk Bi. As in Table III, the subscripts $\perp$ or $\|$ denote the applied magnetic field perpendicular or parallel to the film plane.

\begin{tabular}{lcc}
\hline \hline & Hole pocket & Electron pocket \\
\hline$F_{\perp}(T)$ & 6.3 & 6.4 \\
$F_{\|}(T)$ & 20 & 1.2 \\
& & \\
$m_{c \perp}\left(E_{F}\right)\left(m_{0}\right)$ & 0.064 & 0.065 \\
$m_{c \|}\left(E_{F}\right)\left(m_{0}\right)$ & 0.21 & 0.0084 \\
\hline \hline
\end{tabular}


TABLE V. Elastic-scattering parameters obtained for sample $f$, a heterostructure with a 45-nm-thick central Bi layer. The scattering times for electrons in the binary (bisectrix) direction are given by $\tau_{e 1}\left(\tau_{e 2}\right)$, while $l_{e 1}\left(l_{e 2}\right)$ are the corresponding scattering lengths. The elastic-scattering time (length) for holes are denoted by $\tau_{1 h}\left(l_{h}\right)$.

\begin{tabular}{llll}
\hline \hline Electron pocket & $\tau_{t, e 1}(\mathrm{ps})$ & 0.47 & $( \pm 0.05)$ \\
& $\tau_{t, e 2}(\mathrm{ps})$ & 1.9 & $( \pm 0.2)$ \\
& $l_{e 1}(\mu \mathrm{m})$ & 0.38 & $( \pm 0.08)$ \\
& $l_{e 2}(\mu \mathrm{m})$ & 0.10 & $( \pm 0.02)$ \\
Hole pocket & & & \\
& $\tau_{t, h}(\mathrm{ps})$ & 1.1 & $( \pm 0.1)$ \\
& $l_{h}(\mu \mathrm{m})$ & 0.5 & $( \pm 0.1)$ \\
\hline \hline
\end{tabular}

$10^{15} \mathrm{~cm}^{-2}$, a value that is roughly two orders of magnitude larger than the density of high mobility holes or electrons in the film, is surprising. It is, however, possible that these low mobility charges are caused by the barium fluoride substrate. Before the evaporation of the film the substrate was heated to a sufficiently high temperature for the mass spectrometer to detect a small fluorine signal. If fluorine evaporated from the surface, a residue of barium could have remained which then induced a residual conductance channel, parallel with the heterostructure, but along the substrate-buffer-layer interface.

For a parallel applied magnetic field, the first SdH oscillations became observable for $B_{\|} \geq 1.15 \mathrm{~T}$. Using the measured $F_{\|}$and the mass tensor, ${ }^{1,5}$ the diameter of the cyclotron orbit corresponding to this field is $d_{\text {cycle }}=57$ $\mathrm{nm}$. This is close to the Bi-layer thickness $d_{\mathrm{Bi}}=45 \mathrm{~nm}$. We speculate that for smaller magnetic fields $\mathrm{SdH}$ oscillations were not observed because $d_{\text {cycl }}$ becomes larger than the Bi-layer thickness.

The experimentally observed cross section for perpendicular applied magnetic field in the heterostructure with 45-nm-thick central $\mathrm{Bi}$ layer is $F_{\perp}=17 \mathrm{~T}$ (Table III). Comparing this value to $F_{\perp}=8.3 \mathrm{~T}$, obtained on a thicker film (sample $h$ : 500-nm Bi film; see Table I), and to the cross section $F_{\perp}=6.4 \mathrm{~T}$ in bulk $\mathrm{Bi}$, we clearly see the increasing influence of the interface as the film thickness decreases. Furthermore, in heterostructures with identical thicknesses but with different $\mathrm{Sb}$ concentrations $x$, an increase in $F_{\perp}$ with increasing $x$ was observed, indicated that the band bending at the $\mathrm{Bi}_{1-x} \mathrm{Sb}_{x} / \mathrm{Bi}$ interface became stronger. If we assume that the effective masses in a thin film remain the same as in bulk, then our result $F_{\perp}=17 \mathrm{~T}$ for the 45-nm-thick Bi layer indicates that the electron pockets as well as the hole pocket are scaled up by a factor $\sqrt{17 / 6.4}$ in all three dimensions. From this we expect an enhanced sheet carrier density (with respect to bulk values $)$ of $(17 / 6.4)^{1.5}\left(n_{\text {bulk }} / d_{\mathrm{Bi}}\right)=5.4 \times 10^{12}$ $\mathrm{cm}^{-2}$. Indeed, this value is in very reasonable agreement with $N_{2}=7.7 \times 10^{12} \mathrm{~cm}^{-2}$, the fit result given in Table II.

In a thin film with a Fermi wavelength comparable to and an elastic-scattering length larger than the film thickness, quantum confinement results in discrete, twodimensional conduction bands. The contribution $N_{2,2 D}$ of the lowest band to the sheet density $N_{2}$ is obtained from the SdH period $F$ through the relation $N_{2,2 D}=6(e / h) F^{32}$ The factor 6 results from the spin degeneracy in the three electron pockets. From the measured $\mathrm{SdH}$ period we find $N_{2,2 D}=(2.5 \pm 0.3) \times 10^{12} \mathrm{~cm}^{-2}$. This value is a factor of 3 smaller than the result $N_{2}=7.7 \times 10^{12} \mathrm{~cm}^{-2}$ obtained from our fits, which suggests that only the lowest three conduction bands were occupied.

We can compare our results to those from other groups. The thinnest epitaxial film described by Partin et al. ${ }^{6}$ had $d_{\mathrm{Bi}}=100 \mathrm{~nm}, n=9 \times 10^{17} \mathrm{~cm}^{-3}$ and $\mu_{1}=1.0 \times 10^{5} \mathrm{~cm}^{2} / \mathrm{V}$ s. This film did not exhibit SdH oscillations. Translating the experimentally obtained sheet carrier density $\mathrm{N}_{2}$ into a volume density, we find $n=17 \times 10^{17} \mathrm{~cm}^{-3}$ for our heterostructure sample $f$. Epitaxial $\mathrm{Bi}$ films on mica have been described by Jing et al. ${ }^{12}$ This group obtained films of $30-\mathrm{nm}$ thickness with a rms roughness of $0.82 \mathrm{~nm}$ and, because of twinning, crystallites with sizes of about $0.5 \mu \mathrm{m}$. The high value for the resistivity, $\rho_{4.2 \mathrm{~K}}=400 \mu \Omega \mathrm{cm}$, in these films is most likely due to the twin boundaries. SdH oscillations have previously been described in detail for bulk $\mathbf{B i}$, but so far there have only been two reported observations in thin Bi films. ${ }^{5,6}$ The reported $\mathrm{SdH}$ oscillation period, for a magnetic field perpendicular to the film plane, corresponds to $F \approx 1.4 \mathrm{~T} .{ }^{5}$ Even though in these samples the preferred growth direction had a trigonal axis perpendicular to the substrate, this value for $F$ is a factor of 10 smaller than the one measured in our films. Since $F \approx 1.4$ $T$ is close to the value expected for the binary-trigonal cross section of the electron pocket, the existence of polycrystalline regions in the samples of Ref. 5 is likely.

In $\mathrm{CdTe} / \mathrm{Bi} / \mathrm{CdTe}$ heterostructures with Bi-layer thicknesses between 20 and $40 \mathrm{~nm}$, Hoffman et al. ${ }^{7}$ found that the low-temperature majority carriers are holes with densities $p \gg n$. Their data are in accord with the observation by Komnik and co-workers ${ }^{7,8}$ that the carrier density in the thin-film limit exceeds the intrinsic, bulk value by an amount $P_{2} / d$, where $d$ is the film thickness and $P_{2}$

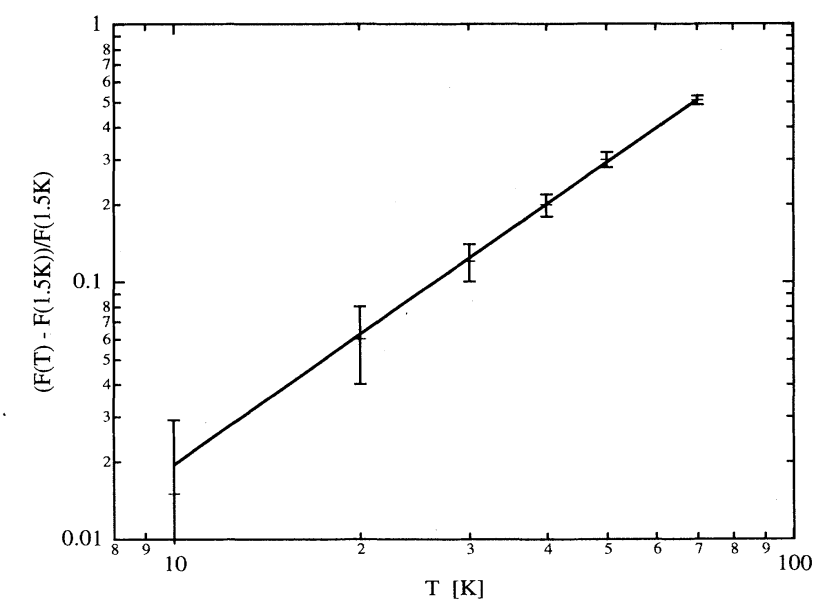

FIG. 8. Relative change of $F$ (the inverse of the SdHoscillation period) with temperature. The straight line on this plot corresponds to a power law $T^{\alpha}$ with $\alpha=1.7$. 
( $\approx 8 \times 10^{12} \mathrm{~cm}^{-2}$ for the data by Hoffman et al.) is a sheet density associated with surface states. It is clear, however, that this surface-state density must depend sensitively on the details of the film interfaces. Our results for $\mathrm{Bi}_{1-x} \mathrm{Sb}_{x} / \mathrm{Bi} / \mathrm{Bi}_{1-x} \mathrm{Sb}_{x}$ heterostructures show that a much more bulklike behavior, $n \approx p$, of nearly equal electron and hole concentrations is possible, certainly as far as the high mobility carriers are concerned.

Finally, we remark on the small but significant change in the SdH oscillation period with increasing temperature, as seen in Fig. 7(b). Empirically, we can associate this behavior with a temperature-dependent $F(T)$. In Fig. 8 we plot the change in $F(T)$ relative to its lowtemperature value $F(T=1.5 \mathrm{~K})$. Remarkably, over the whole temperature range studied, we observe a power-law behavior

$$
\frac{F(T)-F(T=1.5 \mathrm{~K})}{F(T)=1.5 \mathrm{~K})} \sim T^{\alpha}
$$

From Fig. 8 we obtain an exponent $\alpha \approx 1.7$. This power law appears to reflect an expansion of the extremal orbits at the Fermi surface with increasing temperature.

\section{SUMMARY AND CONCLUSIONS}

We have described a process to fabricate thin, epitaxial Bi films of arbitrary thickness. This process is based on MBE growth of $\mathrm{Bi}$ on $\mathrm{Bi}_{1-x} \mathrm{Sb}_{x}$ buffer layer, and has several advantages over previous approaches that deposited $\mathrm{Bi}$ directly on either mica or $\mathrm{BaF}_{2}$. By sandwiching a central $\mathrm{Bi}$ film between semiconducting $\mathrm{Bi}_{1-x} \mathrm{Sb}_{x}$ buffers, a high carrier mobility is achieved as well as a clean termination of the film surfaces with little band bending. An additional benefit is the protection from oxidation. The method is easily extendable to multilayer fabrication. We discussed RHEED, SEM, and AFM, as well as transport measurements in detail for a $90-\mathrm{nm}$ $\mathrm{Bi}_{0.95} \mathrm{Sb}_{0.05} / 45-\mathrm{nm} \mathrm{Bi} / 65-\mathrm{nm} \mathrm{Bi}_{0.95} \mathrm{Sb}_{0.05}$ heterostructure. The single crystallinity of the structure and high quality of the $\mathrm{Bi} / \mathrm{Bi}_{0.95} \mathrm{Sb}_{0.05}$ interfaces resulted in a high transport mobility, low bulklike carrier density, and clearly observable $\mathrm{SdH}$ oscillations. At helium temperatures there is only conductance in the $\mathrm{Bi}$ layer, because the parallel conductance in the $\mathrm{Bi}_{0.95} \mathrm{Sb}_{0.05}$ is almost frozen out due its semiconducting behavior. The experimental results for the thin $\mathrm{Bi}$ layer show nearly equal densities of high mobility electrons and holes that are enhanced over the bulk values for $\mathrm{Bi}$ by a factor of the order 6 . For magnetic fields parallel to the film plane the observed SdH oscillations could be associated with electron pockets. For a perpendicular magnetic field a distinction between electron or hole pockets was not possible. The observed long elastic-scattering lengths $\left(l_{e 1}=0.38 \mu \mathrm{m}\right.$, $\left.l_{h}=0.5 \mu \mathrm{m}\right)$, together with the fact that only a few twodimensional states are occupied in a 45-nm-thick Bi layer, should make $\mathrm{Bi} / \mathrm{Bi}_{0.95} \mathrm{Sb}_{0.05}$ heterostructures highly suitable candidates for a further investigation of mesoscopic phenomena. The fabrication process described here can easily be extended to allow not only for a tight control of the Bi-layer thickness, but also to tune the interface characteristics and quality. By changing the growth temperature, e.g., surface roughness can be introduced in a controlled way. Several recent theoretical approaches include a surface roughness parameter since it controls the scattering processes in the size quantization regime. Additional possibilities arise by patterning $\mathrm{Bi} / \mathrm{Bi}_{0.95} \mathrm{Sb}_{0.05}$ heterostructures with $e$-beam lithography to study mesoscopic phenomena in submicron, single crystalline systems. Investigations on two such systems, namely lateral point contacts and in-plane gated devices, are currently in progress.

\section{ACKNOWLEDGMENTS}

We wish to thank Dick van der Marel, Ronald Griessen, Rinke Wijngaarden, Bart Geerligs, and Ozkar Schannen for their help with this project. We have also benefited from stimulating discussions with Jaap Caro, Mei Lu, and Rena Zeive. This work was part of the research program of the Stichting Fundamenteel Onderzoek der Materie (FOM), which is financially supported by the Nederlandse Organisatie voor Wetenschappelijk Onderzoek (NWO). The work at the University of Chicago was supported in part by the MRSEC Program of the National Science Foundation under Award Number DMR-9400379. H.M.J. thanks the Alfred P. Sloan Foundation and the David and Lucile Packard Foundation for fellowship support.
*Permanent address: James Franck Institute and Department of Physics, The University of Chicago, Chicago, IL 60637.

${ }^{1}$ G. E. Smith, G. A. Baraff, and J. M. Rowell, Phys. Rev. 135, A1118 (1964).

${ }^{2}$ E. S. Itsekevich and L. M. Fisher, Zh. Eksp. Teor. Fiz. 53, 92 (1967) [Sov. Phys. JETP 26, 66 (1968)].

${ }^{3}$ G. E. Smith, L. C. Hebel, and S. J. Buchsbaum, Phys. Rev. 129, 154 (1963).

${ }^{4}$ H. T. Chu, J. Phys. Chem Solids 48, 845 (1987).

${ }^{5}$ H. T. Chu, P. N. Henriksen, Jing Jing, Hong Wang, and Xiaofeng Xu, Phys. Rev. B 45, 11233 (1992).

${ }^{6}$ D. L. Partin, J. Heremans, D. T. Morelli, C. M. Thrush, C. H. Olk, and T. A. Perry, Phys. Rev. B 38, 3818 (1988).

${ }^{7}$ C. A. Hoffman, J. R. Meyer, F. J. Bartolli, A. Di Venere, X. J.
Yi, C. L. Hou, H. C. Wang, J. B. Ketterson, and G. K. Wong, Phys. Rev. B 48, 11431 (1993).

${ }^{8}$ Yu. F. Komnik, E. I. Bukhshtab, Yu. V. Nikitin, and V. V. Andrievskii, Zh. Eksp. Teor. Fiz. 60, 669 (1971) [Sov. Phys. JETP 33, 364 (1971)].

${ }^{9}$ Yu. F. Komnik and V. V. Andrievskii, Fiz. Nizk. Temp. 1, 104 (1975) [Sov. J. Low Temp. Phys. 1, 51 (1975)].

${ }^{10}$ V. P. Duggal and Raj Rup, J. Appl. Phys. 40, 492 (1969).

${ }^{11}$ B. Y. Jin, H. K. Wong, G. K. Wong, J. B. Ketterson, and Y. Eckstein, Thin Solid Films 110, 29 (1983).

12 Jing Jing, P. N. Henriksen, H. T. Chu, and Hong Wang, Appl. Surf. Sci. 62, 105 (1992).

${ }^{13}$ D. L. Partin, C. M. Thrush, J. Heremans, D. T. Morelli, and C. H. Olk, J. Vac. Sci. Technol. B 7, 348 (1988). 
${ }^{14}$ D. T. Morelli, D. L. Partin, and J. Heremans, Semicond. Sci. Technol. 5, S257 (1990).

15J. Takahashi and T. Miyagawa, Jpn. J. Appl. Phys. 31, L1114 (1992).

${ }^{16}$ S. Nahm, L. Salamanca-Riba, D. L. Partin, and J. Heremans, J. Mater. Res. 5, 784 (1990).

${ }^{17}$ P. Cucka, and C. S. Barrett, Acta Crystallogr. 15, 865 (1962).

${ }^{18}$ A. L. Jain, Phys. Rev. 114, 1518 (1959).

${ }^{19}$ S. Golin, Phys. Rev. 176, 830 (1968).

${ }^{20}$ D. M. Brown and S. J. Silverman, Phys. Rev. 136, A290 (1964).

${ }^{21}$ O. P. Hansen, I. F. I. Mikhail, M. Yu. Larenyuk, and N. Ya. Minina, J. Low Temp. Phys. 95, 481 (1994).

${ }^{22}$ Quartz \& Silice, Nemours, France.

${ }^{23}$ R. M. Overney, H. Haefke, E. Meyer, and H.-J. Güntherodt, Surf. Sci. Lett. 277, L29 (1992).

${ }^{24}$ M. Prutton, Surface Physics, 2nd ed. (Oxford University Press, Oxford, 1983), p. 49.

${ }^{25}$ M. J. Joshi, and B. S. Shah, Cryst. Def. Amorph. Mater. 11,
229 (1985).

${ }^{26}$ R. Hartman, Phys. Rev. 181, 1070 (1969).

${ }^{27}$ M. J. Kane, N. Apsley, D. A. Anderson, L. L. Taylor, and T. Kerr, J. Phys. C 18, 5629 (1985).

${ }^{28}$ M. A. Reed, W. P. Kirk, and P. S. Kobiela, IEEE J. Quantum Electron. QE-22, 1753 (1986).

${ }^{29}$ E. F. Schubert, K. Ploog, H. Dämbkes, and K. Heime, Appl. Phys. A 33, 63 (1984).

${ }^{30}$ L. M. Roth, and P. N. Argyres, in Semiconductors and Semimetals, edited by R. K. Willardson and A. C. Beer (Academic, New York, 1966), Vol. 1, p. 159.

${ }^{31}$ S. Das Sarma, Phys. Rev. B 32, 8442 (1985).

${ }^{32}$ C. Kittel, Introduction to Solid State Physics, 6th ed. (Wiley, New York, 1986), Chap. 9.

${ }^{33}$ W. Joss, R. Griessen, and E. Fawcett, in Electron States and Fermi Surfaces of Strained Elements, edited by K.-H. Hellwege and J. L. Olsen, Landolt-Börnstein, New Series, Group III, Vol. 13, Pt. b (Springer-Verlag, Berlin, 1983), p. 46. 


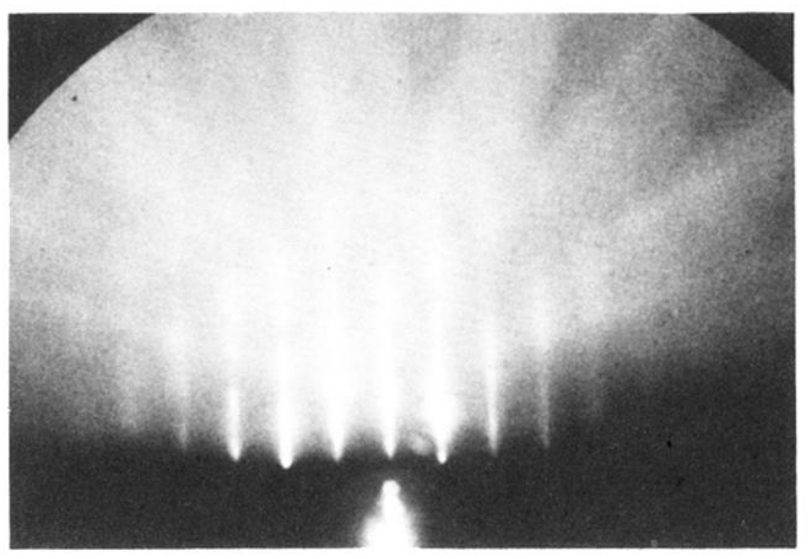

FIG. 2. RHEED pattern of a 675-nm $\mathrm{Bi}(111)$ film grown at $110^{\circ} \mathrm{C}$ (sample c). The electron beam had an energy of $50 \mathrm{keV}$ and a $(\overline{1}, 0,1)$ azimuth orientation. The diffraction pattern exhibits 2D character (lines) with only a small additional 3D component (spots), indicative of a very smooth surface. 


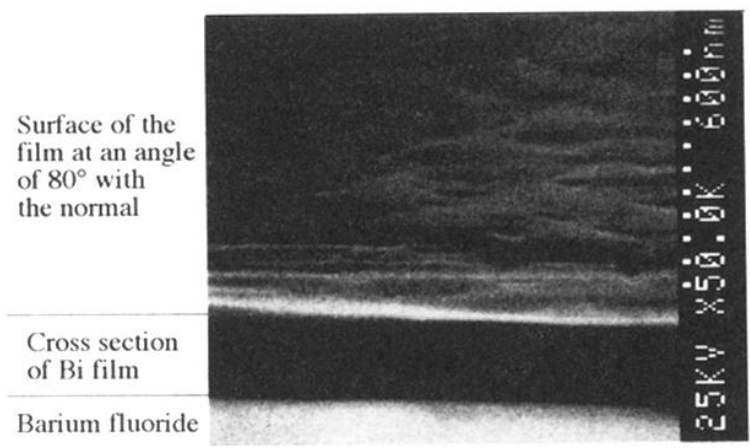

FIG. 3. Scanning-electron-microscope (SEM) image of a 200-nm-thick heterostructure (sample $f$ ). A cross section of the heterostructure is seen in the foreground, with very little surface roughness visible at the top (magnification $50000 \times$ at a tilt angle of $80^{\circ}$ with respect to the surface normal). 


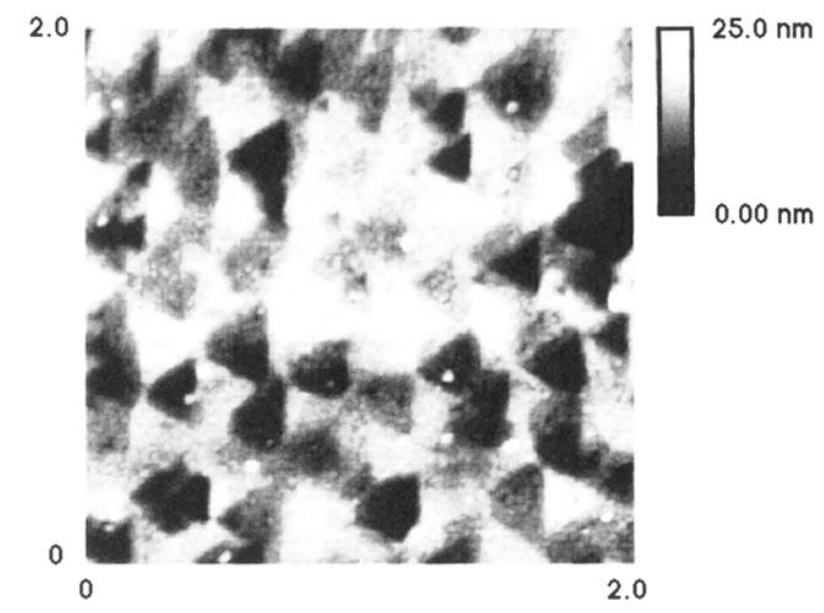

FIG. 4. Atomic-force-microscope (AFM) image of the sample sample as in Fig. 3 (sample $f$ ) after a selective etch for dislocations. The scan field is $2 \times 2 \mu \mathrm{m}^{2}$ and is representative of the whole film. All triangular pits exhibit the same orientation, indicating that the sample is single crystalline. The etch pit density corresponds to $2 \times 10^{9}$ dislocations per $\mathrm{cm}^{2}$. 